\title{
ON NEW GENERALIZATIONS OF HILBERT-PACHPATTE TYPE INTEGRAL INEQUALITIES
}

\author{
LÜ ZHONGXUE
}

\begin{abstract}
In this paper, some new generalizations of Hilbert-Pachpatte type inequalities are given by introducing some parameters $r_{i}, i \in\{1,2, \ldots, n\}$.
\end{abstract}

\section{Introduction}

Hilbert's double series inequality and its integral version [3, Theorem 316] have been generalized in several directions (see [1-6,8,9]). Pachpatte [10-12] considered inequalities similar to those of Hilbert. Recenty, G. D. Handley, J. I. Koliha and J. E. Pečarič [13] considered a new class of Hilbert-Pachpatte type integral inequalities by specializing the parameters and the functions $\Phi_{i}$.

In this paper, we show some new generalizations of Hilbert-Pachpatte type integral inequalities in [13] by introducing some parameters.

\section{Notation and Preliminaries}

The symbols $N, Z, R$ have their usual meaning; $R_{+}$denotes the interval $[0, \infty)$. The following notation and hypotheses will be used throughout the paper:

$$
\begin{aligned}
& I=\{1, \ldots, n\} \quad n \in N \\
& m_{i}, i \in I \quad m_{i} \in N \\
& k_{i}, i \in I \quad k_{i} \in\left\{0, \ldots, m_{i}-1\right\} \\
& x_{i}, i \in I \quad x_{i} \in R, x_{i}>0 \\
& p_{i}, q_{i}, r_{i} \in I \quad p_{i}, q_{i}, r_{i} \in R_{+}, 1 / p_{i}+1 / q_{i}=1-1 / r_{i} \\
& p, q, r \quad 1 / p=\sum_{i=1}^{n}\left(1 / p_{i}\right), \quad 1 / q+1 / r=\sum_{i=1}^{n}\left(1 / q_{i}+1 / r_{i}\right) \\
& a_{i}, b_{i}, i \in I \quad a_{i}, b_{i} \in R_{+}, a_{i}+b_{i}=1 \\
& w_{i}, i \in I \quad w_{i} \in R, w_{i}>0, \quad \sum_{i=1}^{n} w_{i}=1 \\
& \alpha_{i}, i \in I \quad \alpha_{i}=\left(a_{i}+b_{i} \frac{q_{i} r_{i}}{q_{i}+r_{i}}\right)\left(m_{i}-k_{i}-1\right) \\
& \beta_{i}, i \in I \quad \beta_{i}=a_{i}\left(m_{i}-k_{i}-1\right) \\
& u_{i}, i \in I \quad u_{i} \in C^{m_{i}^{\prime}}\left(\left[0, x_{i}\right]\right) \text { for some } m_{i}^{\prime} \geq m_{i} \\
& \Phi_{i}, i \in I \quad \Phi_{i} \in C^{1}\left(\left[0, x_{i}\right]\right), \quad \Phi_{i} \geq 0 .
\end{aligned}
$$

Received January 4, 2002; revised February 21, 2001.

2000 Mathematics Subject Classification. 26D15.

Key words and phrases. Inequalities, Hilbert-Pachpatte inequalities, Hölder inequality. 
Here the $u_{i}$ are given functions of sufficient smoothness, and the $\Phi_{i}$ are given continuous nonnegative functions.

\section{Statement of Results}

Our main result is given in the following theorem.

Theorem 3.1. Let $u_{i} \in C^{m_{i}}\left(\left[0, x_{i}\right]\right)$. for $i \in I$. If

$$
\left|u_{i}^{\left(k_{i}\right)}\left(s_{i}\right)\right| \leq \int_{0}^{s_{i}}\left(s_{i}-\tau_{i}\right)^{m_{i}-k_{i}-1} \Phi_{i}\left(\tau_{i}\right) d \tau_{i} \quad s_{i} \in\left[0, x_{i}\right], \quad i \in I
$$

Then

$$
\begin{aligned}
& \int_{0}^{x_{1}} \cdots \int_{0}^{x_{n}} \frac{\prod_{i=1}^{n}\left|u_{i}^{\left(k_{i}\right)}\left(s_{i}\right)\right|}{\sum_{i=1}^{n} w_{i} s_{i}^{\left(\alpha_{i}+1\right) \frac{q_{i}+r_{i}}{q_{i} r_{i} w_{i}}}} d s_{1} \cdots d s_{n} \\
\leq & U \prod_{i=1}^{n} x_{i}^{\frac{q_{i}+r_{i}}{q_{i} r_{i}}} \prod_{i=1}^{n}\left(\int_{0}^{x_{i}}\left(x_{i}-s_{i}\right)^{\beta_{i}+1} \Phi_{i}\left(s_{i}\right)^{p_{i}} d s_{i}\right)^{1 / p_{i}}
\end{aligned}
$$

where

$$
U=\frac{1}{\prod_{i=1}^{n}\left[\left(\alpha_{i}+1\right)^{\frac{q_{i}+r_{i}}{q_{i} r_{i}}}\left(\beta_{i}+1\right)^{1 / p_{i}}\right]}
$$

Proof. Factorize the integrand on the right side of (3.1) as

$$
\left(s_{i}-\tau_{i}\right)^{\left(a_{i} \frac{p_{i}-1}{p_{i}}+b_{i}\right)\left(m_{i}-k_{i}-1\right)} \times\left(s_{i}-\tau_{i}\right)^{\left(a_{i} / p_{i}\right)\left(m_{i}-k_{i}-1\right)} \Phi_{i}\left(\tau_{i}\right)
$$

and apply Hölder's inequality [7, p.106]. Then

$$
\begin{aligned}
\left|u_{i}^{\left(k_{i}\right)}\left(s_{i}\right)\right| & \leq\left(\int_{0}^{s_{i}}\left(s_{i}-\tau_{i}\right)^{\left(a_{i}+b_{i} \frac{q_{i} r_{i}}{q_{i}+r_{i}}\right)\left(m_{i}-k_{i}-1\right)} d \tau_{i}\right)^{1-1 / p_{i}}\left(\int_{0}^{s_{i}}\left(s_{i}-\tau_{i}\right)^{a_{i}\left(m_{i}-k_{i}-1\right)} \Phi_{i}\left(\tau_{i}\right)^{p_{i}} d \tau_{i}\right)^{1 / p_{i}} \\
& =\frac{s_{i}^{\left(\alpha_{i}+1\right) \frac{q_{i}+r_{i}}{q_{i} r_{i}}}}{\left(\alpha_{i}+1\right)^{\frac{q_{i}+r_{i}}{q_{i} r_{i}}}}\left(\int_{0}^{s_{i}}\left(s_{i}-\tau_{i}\right)^{\beta_{i}} \Phi_{i}\left(\tau_{i}\right)^{p_{i}} d \tau_{i}\right)^{1 / p_{i}}
\end{aligned}
$$

Using the inequality of means $[7, \mathrm{p} .15]$

$$
\prod_{i=1}^{n} s_{i}^{\left(\alpha_{i}+1\right) \frac{q_{i}+r_{i}}{q_{i} r_{i}}} \leq \sum_{i=1}^{n} w_{i} s_{i}^{\left(\alpha_{i}+1\right) \frac{q_{i}+r_{i}}{q_{i} r_{i} w_{i}}}
$$

we get

$$
\prod_{i=1}^{n}\left|u_{i}^{\left(k_{i}\right)}\left(s_{i}\right)\right| \leq W \sum_{i=1}^{n} w_{i} s_{i}^{\left(\alpha_{i}+1\right) \frac{q_{i}+r_{i}}{q_{i} r_{i} w_{i}}} \prod_{i=1}^{n}\left(\int_{0}^{s_{i}}\left(s_{i}-\tau_{i}\right)^{\beta_{i}} \Phi_{i}\left(\tau_{i}\right)^{p_{i}} d \tau_{i}\right)^{1 / p_{i}}
$$


where

$$
W=\frac{1}{\prod_{i=1}^{n}\left(\alpha_{i}+1\right)^{\frac{q_{i}+r_{i}}{q_{i} r_{i}}}}
$$

In the following estimate we apply Hölder's inequality and at the end, change the order of integration:

$$
\begin{aligned}
& \int_{0}^{x_{1}} \cdots \int_{0}^{x_{n}} \frac{\prod_{i=1}^{n}\left|u_{i}^{\left(k_{i}\right)}\left(s_{i}\right)\right|}{\sum_{i=1}^{n} w_{i} s_{i}^{\left(\alpha_{i}+1\right) \frac{q_{i}+r_{i}}{q_{i} r_{i} w_{i}}}} d s_{1} \cdots d s_{n} \\
\leq & W \prod_{i=1}^{n}\left[\int_{0}^{x_{i}}\left(\int_{0}^{s_{i}}\left(s_{i}-\tau_{i}\right)^{\beta_{i}} \Phi_{i}\left(\tau_{i}\right)^{p_{i}} d \tau_{i}\right)^{1 / p_{i}} d s_{i}\right] \\
\leq & W \prod_{i=1}^{n} x_{i}^{\frac{q_{i}+r_{i}}{q_{i} r_{i}}}\left(\int_{0}^{x_{i}}\left(\int_{0}^{s_{i}}\left(s_{i}-\tau_{i}\right)^{\beta_{i}} \Phi_{i}\left(\tau_{i}\right)^{p_{i}} d \tau_{i}\right) d s_{i}\right)^{1 / p_{i}} \\
= & \frac{W^{n}}{\prod^{n}\left(\beta_{i}+1\right)^{1 / p_{i}}} \prod_{i=1}^{\frac{q_{i}+r_{i}}{q_{i} r_{i}}} \prod_{i=1}^{n}\left(\int_{0}^{x_{i}}\left(x_{i}-\tau_{i}\right)^{\beta_{i}+1} \Phi_{i}\left(\tau_{i}\right)^{p_{i}} d \tau_{i}\right)^{1 / p_{i}}
\end{aligned}
$$

This proves the theorem.

Corollary 3.2. Under the assumptions of Theorem 3.1,

$$
\begin{aligned}
& \int_{0}^{x_{1}} \cdots \int_{0}^{x_{n}} \frac{\prod_{i=1}^{n}\left|u_{i}^{\left(k_{i}\right)}\left(s_{i}\right)\right|}{\sum_{i=1}^{n} w_{i} s_{i}^{\left(\alpha_{i}+1\right) \frac{q_{i}+r_{i}}{q_{i} r_{i} w_{i}}}} d s_{1} \cdots d s_{n} \\
\leq & p^{1 / p} U \prod_{i=1}^{n} x_{i}^{\frac{q_{i}+r_{i}}{q_{i} r_{i}}}\left(\sum_{i=1}^{n} \frac{1}{p_{i}} \int_{0}^{x_{i}}\left(x_{i}-s_{i}\right)^{\beta_{i}+1} \Phi_{i}\left(s_{i}\right)^{p_{i}} d s_{i}\right)^{1 / p_{i}}
\end{aligned}
$$

where $U$ is given by (3.3).

Proof. By the inequality of means, for any $A_{i} \geq 0$,

$$
\prod_{i=1}^{n} A_{i}^{1 / p_{i}} \leq p^{1 / p}\left(\sum_{i=1}^{n} \frac{1}{p_{i}} A_{i}\right)^{1 / p}
$$

The corollary then follows from the preceding theorem.

In the following sections we discuss various choices of the functions $\Phi_{i}$. 


\section{The First Inequality}

Theorem 4.1. Let $u_{i} \in C^{m_{i}}\left(\left[0, x_{i}\right]\right)$ be such that $u_{i}^{(j)}(0)=0$ for $j \in\left\{0, \cdots, m_{i}-1\right\}$, $i \in I$. Then

$$
\begin{aligned}
\int_{0}^{x_{1}} \cdots \int_{0}^{x_{n}} \frac{\prod_{i=1}^{n}\left|u_{i}^{\left(k_{i}\right)}\left(s_{i}\right)\right|}{\sum_{i=1}^{n} w_{i} s_{i}^{\left(\alpha_{i}+1\right) \frac{q_{i}+r_{i}}{q_{i} r_{i} w_{i}}}} d s_{1} \cdots d s_{n} \\
\leq U_{1} \prod_{i=1}^{n} x_{i}^{\frac{q_{i}+r_{i}}{q_{i} r_{i}}} \prod_{i=1}^{n}\left(\int_{0}^{x_{i}}\left(x_{i}-s_{i}\right)^{\beta_{i}+1}\left|u_{i}^{\left(m_{i}\right)}\left(s_{i}\right)\right|^{p_{i}} d s_{i}\right)^{1 / p_{i}}
\end{aligned}
$$

where

$$
U_{1}=\frac{1}{\prod_{i=1}^{n}\left[\left(m_{i}-k_{i}-1\right) !\left(\alpha_{i}+1\right)^{\frac{q_{i}+r_{i}}{q_{i} r_{i}}}\left(\beta_{i}+1\right)^{1 / p_{i}}\right]}
$$

Proof. By [10, Eq.(7)],

$$
u_{i}^{\left(k_{i}\right)}\left(s_{i}\right)=\frac{1}{\left(m_{i}-k_{i}-1\right) !} \int_{0}^{s_{i}}\left(s_{i}-\tau_{i}\right)^{m_{i}-k_{i}-1} u_{i}^{\left(m_{i}\right)}\left(\tau_{i}\right) d \tau_{i}
$$

Inequality (4.1) is proved when we set

$$
\Phi_{i}\left(s_{i}\right)=\frac{\left|u_{i}^{\left(m_{i}\right)}\left(s_{i}\right)\right|}{\left(m_{i}-k_{i}-1\right) !}
$$

in Theorem 3.1.

Corollary 4.2. Under the assumptions of Theorem 4.1,

$$
\begin{gathered}
\int_{0}^{x_{1}} \cdots \int_{0}^{x_{n}} \frac{\prod_{i=1}^{n}\left|u_{i}^{\left(k_{i}\right)}\left(s_{i}\right)\right|}{\sum_{i=1}^{n} w_{i} s_{i}^{\left(\alpha_{i}+1\right) \frac{q_{i}+r_{i}}{q_{i} r_{i} w_{i}}}} d s_{1} \cdots d s_{n} \\
\leq p^{1 / p} U_{1} \prod_{i=1}^{n} x_{i}^{\frac{q_{i}+r_{i}}{q_{i} r_{i}}}\left(\sum_{i=1}^{n} \frac{1}{p_{i}} \int_{0}^{x_{i}}\left(x_{i}-s_{i}\right)^{\beta_{i}+1}\left|u_{i}^{\left(m_{i}\right)}\left(s_{i}\right)\right|^{p_{i}} d s_{i}\right)^{1 / p}
\end{gathered}
$$

where $U_{1}$ is given by (4.2). 


\section{The Second Inequality}

Theorem 5.1. Let $u_{i} \in C^{m_{i}+1}\left(\left[0, x_{i}\right]\right)$ be such that $u_{i}^{(j)}(0)=0$ for $j \in\left\{0, \ldots, m_{i}\right\}$, $i \in I$, and let $\rho \in C^{1}([0, \infty))$. Then

$$
\begin{aligned}
\int_{0}^{x_{1}} \cdots \int_{0}^{x_{n}} \frac{\prod_{i=1}^{n}\left|u_{i}^{\left(k_{i}\right)}\left(s_{i}\right)\right|}{\sum_{i=1}^{n} w_{i} s_{i}^{\left(\alpha_{i}+1\right) \frac{q_{i}+r_{i}}{q_{i} r_{i} w_{i}}}} d s_{1} \cdots d s_{n} \\
\leq U_{1} \prod_{i=1}^{n} x_{i}^{\frac{q_{i}+r_{i}}{q_{i} r_{i}}} \prod_{i=1}^{n}\left[\int_{0}^{x_{i}}\left(x_{i}-s_{i}\right)^{\beta_{i}+1} \frac{s_{i}^{p_{i}-1}}{\rho\left(s_{i}\right)^{p_{i}}}\left(\int_{0}^{s_{i}}\left|\left(\rho\left(\sigma_{i}\right) u_{i}^{\left(m_{i}\right)}\left(\sigma_{i}\right)\right)^{\prime}\right|^{p_{i}} d \sigma_{i}\right) d s_{i}\right]^{1 / p_{i}}
\end{aligned}
$$

where $U_{1}$ is given by (4.2).

Proof. By [10, Eq.(14)].

$$
u_{i}^{\left(k_{i}\right)}\left(s_{i}\right)=\frac{1}{m_{i}-k_{i}-1} \int_{0}^{s_{i}}\left(s_{i}-\tau_{i}\right)^{\left(m_{i}-k_{i}-1\right)}\left(\frac{1}{\rho\left(\tau_{i}\right)} \int_{0}^{\tau_{i}}\left(\rho\left(\sigma_{i}\right) u_{i}^{\left(m_{i}\right)}\left(\sigma_{i}\right)\right)^{\prime} d \sigma_{i}\right) d \tau_{i}
$$

By Hölder's inequality,

$$
\int_{0}^{\tau_{i}}\left|\left(\rho\left(\sigma_{i}\right) u_{i}^{\left(m_{i}\right)}\left(\sigma_{i}\right)\right)^{\prime}\right| d \sigma_{i} \leq \tau_{i}^{\frac{q_{i}+r_{i}}{q_{i} r_{i}}}\left(\int_{0}^{\tau_{i}}\left|\left(\rho\left(\sigma_{i}\right) u_{i}^{\left(m_{i}\right)}\left(\sigma_{i}\right)\right)^{\prime}\right|^{p_{i}} d \sigma_{i}\right)^{1 / p_{i}},
$$

and inequality (5.1) hold with

$$
\Phi_{i}\left(\tau_{i}\right)=\frac{1}{\left(m_{i}-k_{i}-1\right) !} \frac{\tau_{i}^{\frac{q_{i}+r_{i}}{q_{i} r_{i}}}}{\rho\left(\tau_{i}\right)}\left(\int_{0}^{\tau_{i}}\left|\left(\rho\left(\sigma_{i}\right) u_{i}^{\left(m_{i}\right)}\left(\sigma_{i}\right)^{\prime}\right)\right|^{p_{i}} d \sigma_{i}\right)^{1 / p_{i}} .
$$

in Theorem 3.1.

Corollary 5.2. Under the assumptions of Theorem 5.1.

$$
\begin{aligned}
& \int_{0}^{x_{1}} \cdots \int_{0}^{x_{n}} \frac{\prod_{i=1}^{n}\left|u_{i}^{\left(k_{i}\right)}\left(s_{i}\right)\right|}{\sum_{i=1}^{n} w_{i} s_{i}^{\left(\alpha_{i}+1\right) \frac{q_{i}+r_{i}}{q_{i} r_{i} w_{i}}}} d s_{1} \cdots d s_{n} \\
& \leq P^{1 / p} U_{1} \prod_{i=1}^{n} x_{i}^{\frac{q_{i}+r_{i}}{q_{i} r_{i}}} \prod_{i=1}^{n}\left[\int_{0}^{x_{i}}\left(x_{i}-s_{i}\right)^{\beta_{i}+1} \frac{s_{i}^{p_{i}-1}}{\rho\left(s_{i}\right)^{p_{i}}}\left(\int_{0}^{s_{i}}\left|\left(\rho\left(\sigma_{i}\right) u_{i}^{\left(m_{i}\right)}\left(\sigma_{i}\right)\right)^{\prime}\right|^{p_{i}} d \sigma_{i}\right) d s_{i}\right]^{1 / p}(5.2)
\end{aligned}
$$

where $U_{1}$ is given by (4.2). 


\section{The Third Inequality}

Theorem 6.1. Let $u_{i} \in C^{2 m_{i}}\left(\left[0, x_{i}\right]\right), \rho \in C^{m}([0, \infty))$ with $m=\max _{i} m_{i}, u_{i}^{(j)}(0)=0$ and $\left(\rho\left(s_{i}\right) u_{i}^{\left(m_{i}\right)}\left(s_{i}\right)\right)^{(j)}=0$ at $s_{i}=0$ for $j \in\left\{0, \ldots, m_{i}-1\right\}, i \in I$. Then

$$
\begin{aligned}
\int_{0}^{x_{1}} \cdots \int_{0}^{x_{n}} \frac{\prod_{i=1}^{n}\left|u_{i}^{\left(k_{i}\right)}\left(s_{i}\right)\right|}{\sum_{i=1}^{n} w_{i} s_{i}^{\left(\alpha_{i}+1\right) \frac{q_{i}+r_{i}}{q_{i} r_{i} w_{i}}} d s_{1} \cdots d s_{n}} \\
\leq U_{2} \prod_{i=1}^{n} x_{i}^{\frac{q_{i}+r_{i}}{q_{i} r_{i}}} \prod_{i=1}^{n}\left[\int_{0}^{x_{i}}\left(x_{i}-s_{i}\right)^{\beta_{i}+1} \frac{s_{i}^{m_{i} p_{i}-1}}{\rho\left(s_{i}\right)^{p_{i}}}\left(\int_{0}^{s_{i}}\left|\left(\rho\left(\sigma_{i}\right) u_{i}^{\left(m_{i}\right)}\left(\sigma_{i}\right)\right)^{\left(m_{i}\right)}\right|^{p_{i}} d \sigma_{i}\right) d s_{i}\right]^{1 / p_{i}}(6.1)
\end{aligned}
$$

where

$$
U_{2}=\frac{1}{\prod_{i=1}^{n}\left[\left(m_{i}-1\right) !\left(m_{i}-k_{i}-1\right) !\left(\frac{p_{i} m_{i}-1}{p_{i}-1}\right)^{\frac{q_{i}+r_{i}}{q_{i} r_{i}}}\left(\alpha_{i}+1\right)^{\frac{q_{i}+r_{i}}{q_{i} r_{i}}}\left(\beta_{i}+1\right)^{1 / p_{i}}\right]}
$$

Proof. By [10, Eq.(21)].

$$
\begin{aligned}
u_{i}^{\left(k_{i}\right)}\left(s_{i}\right)= & \frac{1}{\left(m_{i}-1\right) !\left(m_{i}-k_{i}-1\right) !} \\
& \times \int_{0}^{s_{i}}\left(s_{i}-\tau_{i}\right)^{\left(m_{i}-k_{i}-1\right)}\left(\frac{1}{\rho\left(\tau_{i}\right)} \int_{0}^{\tau_{i}}\left(\tau_{i}-\sigma_{i}\right)^{m_{i}-1}\left(\rho\left(\sigma_{i}\right) u_{i}^{\left(m_{i}\right)}\left(\sigma_{i}\right)\right)^{\left(m_{i}\right)} d \sigma_{i}\right) d \tau_{i}
\end{aligned}
$$

For brevity write

$$
F_{i}\left(\sigma_{i}\right)=\left|\left(\rho\left(\sigma_{i}\right) u_{i}^{\left(m_{i}\right)}\left(\sigma_{i}\right)\right)^{\left(m_{i}\right)}\right| .
$$

By Hölder's inequality,

$$
\begin{aligned}
\int_{0}^{\tau_{i}}\left(\tau_{i}-\sigma_{i}\right)^{m_{i}-1} F_{i}\left(\sigma_{i}\right) d \sigma_{i} & \leq\left(\int_{0}^{\tau_{i}}\left(\tau_{i}-\sigma_{i}\right)^{\frac{q_{i} r_{i}\left(m_{i}-1\right)}{q_{i}+r_{i}}} d \sigma_{i}\right)^{1-1 / p_{i}}\left(\int_{0}^{\tau_{i}} F_{i}\left(\sigma_{i}\right)^{p_{i}} d \sigma_{i}\right)^{1 / p_{i}} \\
& =\frac{\tau_{i}^{m_{i}-1 / p_{i}}}{\left(\frac{p_{i} m_{i}-1}{p_{i}-1}\right)^{\frac{q_{i}+r_{i}}{q_{i} r_{i}}}}\left(\int_{0}^{\tau_{i}} F_{i}\left(\sigma_{i}\right)^{p_{i}} d \sigma_{i}\right)^{1 / p_{i}}
\end{aligned}
$$

and inequality (6.1) hold with

$$
\Phi_{i}\left(\tau_{i}\right)=W \frac{\tau_{i}^{m_{i}-1 / p_{i}}}{\rho\left(\tau_{i}\right)}\left(\int_{0}^{\tau_{i}}\left|\left(\rho\left(\sigma_{i}\right) u_{i}^{\left(m_{i}\right)}\left(\sigma_{i}\right)\right)^{\left(m_{i}\right)}\right|^{p_{i}} d \sigma_{i}\right)^{1 / p_{i}} .
$$

where

$$
W=\frac{1}{\left(m_{i}-1\right) !\left(m_{i}-k_{i}-1\right) !\left(\frac{p_{i} m_{i}-1}{p_{i}-1}\right)^{\frac{q_{i}+r_{i}}{q_{i} r_{i}}}}
$$


in Theorem 3.1.

Corollary 6.2. Under the assumptions of Theorem 6.1 .

$$
\begin{aligned}
& \int_{0}^{x_{1}} \cdots \int_{0}^{x_{n}} \frac{\prod_{i=1}^{n}\left|u_{i}^{\left(k_{i}\right)}\left(s_{i}\right)\right|}{\sum_{i=1}^{n} w_{i} s_{i}^{\left(\alpha_{i}+1\right) \frac{q_{i}+r_{i}}{q_{i} r_{i} w_{i}}}} d s_{1} \cdots d s_{n} \\
\leq & p^{1 / p} U_{2} \prod_{i=1}^{n} x_{i}^{\frac{q_{i}+r_{i}}{q_{i} r_{i}}}\left[\sum_{i=1}^{n} \int_{0}^{x_{i}} \frac{\left(x_{i}-s_{i}\right)^{\beta_{i}+1} s_{i}^{m_{i} p_{i}-1}}{p_{i} \rho\left(s_{i}\right)^{p_{i}}} \int_{0}^{s_{i}}\left|\left(\rho\left(\sigma_{i}\right) u_{i}^{\left(m_{i}\right)}\left(\sigma_{i}\right)\right)^{\left(m_{i}\right)}\right|^{p_{i}} d \sigma_{i} d s_{i}\right]^{1 / p},
\end{aligned}
$$

where $U_{2}$ is given by (6.2).

Remark. Let $r_{i} \rightarrow \infty, i \in\{1, \ldots, n\},(3.2),(3.4),(4.1),(4.4),(5.1),(5.2),(6.1)$ and (6.3) change into $(3.2),(3.4),(4.1),(4.4),(5.1),(5.2),(6.1)$ and (6.3) in [13], respectively. Hence (3.2), (3.4), (4.1), (4.4), (5.1), (5.2), (6.1) and (6.3) are generalizations of HilbertPachpatte inequalities in [13].

\section{References}

[1] Yang Bicheng, On Hilbert's integral inequality, J. Math. Anal. Appl. 220(1998), 778-785.

[2] Y. C. Chow, On inequalities of Hilbert and Widder, J. London Math. Soc. 14(1939), 151-154.

[3] G. H. Hary, J. E. Littlewood and G. Polya, Inequalities, Cambridge Univ. Press, London, 1952.

[4] Mingzhe Gao, An improvement of Hardy-Riesz's extension of the Hilbert inequality, J. Math. Res. Exposition 14(1994), 255-259.

[5] Mingzhe Gao, On Hilbert's inequality and its applications, J. Math. Anal. Appl. 212(1997).

[6] D. S. Mitrinović and J. E. Pečaricć, On inequalities of Hilbert and Widder, Proc. Edinburgh. Math. Soc. 34(1991), 411-414.

[7] D. S. Mitrinović and J. E. Pečarić and A. M. Fink. Classial and New Inequalities in Analysis, Kluwer Academic, Dordrecht, 1993.

[8] B. G. Pachpatte, A note on Hilbert type inequality, Tamkang J. Math. 29(1998), 293-298.

[9] B. G. Pachpatte, On some new inequalities similar to Hilbert's inequality, J. Math. Anal. Appl. 226(1998), 166-179.

[10] B. G. Pachpatte, Inequalities similar to the integral analogue of Hilbert's inequality, Tamkang J. Math. 30(1999), 136-146.

[11] B. G. Pachpatte, Inequalities similar to certain extension of Hilbert's inequality, J. Math. Anal. Appl. 243(2000), 217-227.

[12] B. G. Pachpatte, A note on inequality of Hilbert type, Demonstratio Math, in press.

[13] G. D. Handley and J. J. Koliha, New Hilbert-Pachpatte type integral inequalities, J. Math. Anal. Appl. 257(2001), 238-250.

Department of Basic Science of Technology College Xuzhou Normal University, 221011, People's Republic of China.

E-mail: lvzx1@163.net 